\title{
Adolescent major depressive disorder may predict poor psychosocial functioning in young adulthood
}

Lewinsohn PM, Rohde P, Seeley JR, et al. Psychosocial functioning of young adults who have experienced and recovered from major depressive disorder during adolescence. J Abnorm Psychol 2003;112:353-63.

\section{Is adolescent major depressive disorder (MDD) associated with difficulties in young adult psychosocial functioning?}

\section{METHODS}

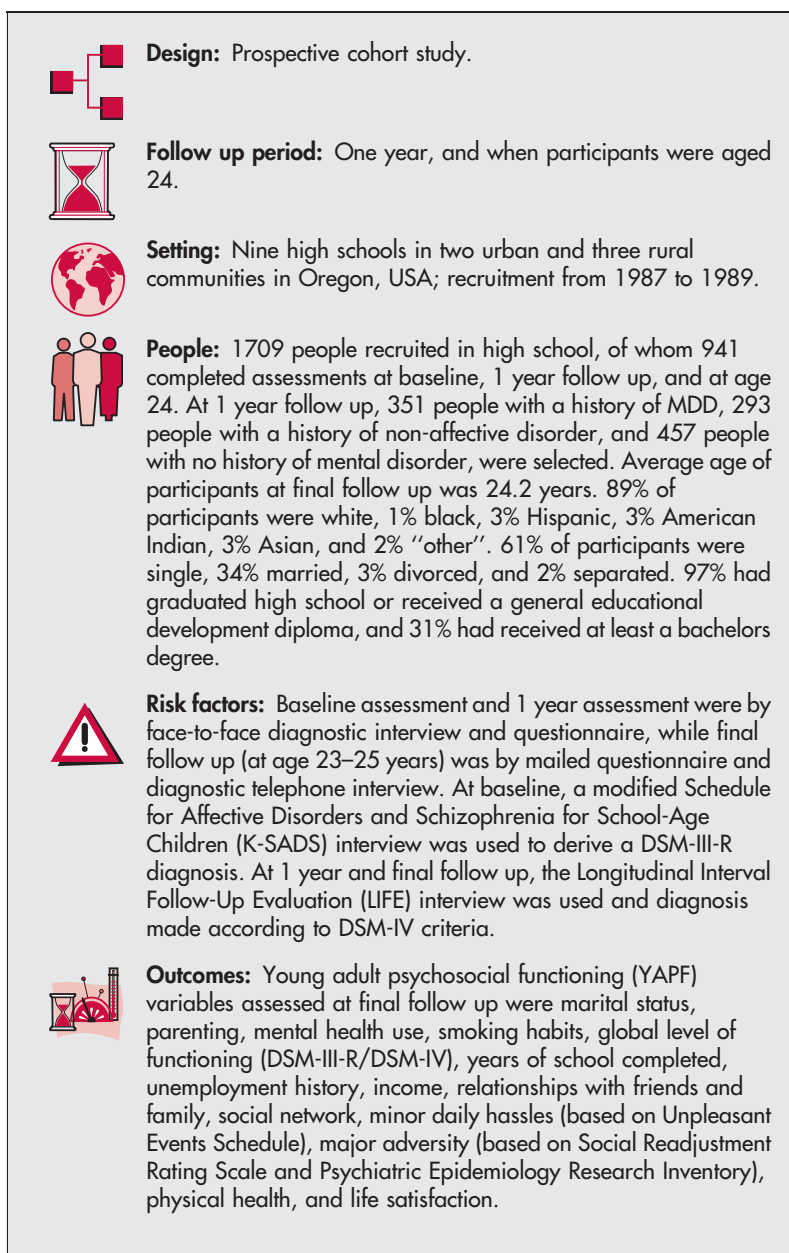

\section{MAIN RESULTS}

Adolescent MDD was significantly associated with low global functioning $(\mathrm{p}<0.001)$, low quality of relations with family
For correspondence: Peter $M$ Lewinsohn, Oregon Research Institute, Franklin Boulevard, Eugene, Oregon, USA; pete@ori.org

Source of funding: This study was funded in part by the National Institute of Mental Health. $(\mathrm{p}<0.01)$, small social network $(\mathrm{p}<0.01)$, minor daily hassles $(\mathrm{p}<0.001)$, major adversity $(\mathrm{p}<0.001)$, low life satisfaction $(\mathrm{p}<0.001)$, and mental health treatment use (OR 1.78, 99\% CI 1.11 to 2.87; analyses adjusted for comorbid non-affective disorder and demographic variables significantly associated with adolescent MDD). Low life satisfaction was the only YAPF variable more strongly associated with adolescent MDD than adolescent nonaffective disorder $(\mathrm{p}<0.001)$. After further adjustment for stability of psychosocial functioning, and depressive symptomatology at the time of young adult assessment, no significant association remained between adolescent MDD and any YAPF measure. 24 participants who did not complete the mailed questionnaire were excluded from analysis.

\section{CONCLUSIONS}

Young adults who have experienced an episode of adolescent MDD experience impairments in many areas of psychosocial functioning.

\section{Commentary}

D uring and around the time of the episode, adolescent major depression is associated with psychological suffering, impairment in social roles, and increased risk for suicide. In this paper, Lewinsohn and colleagues show that adolescents with depression have poorer psychosocial functioning than never depressed adolescents - as reviewed in their article, this exactly parallels similar findings in other studies. The impairment shown in this work was broad, spanning occupational performance, interpersonal functioning, quality of life, and physical well being. The important contribution of this particular paper is in the examination of the specificity of this relationship and of alternative causal models.

In their examination, most of the psychosocial impairment appears to be associated with psychopathology in general and not with depression per se. While this perhaps should come as no surprise, the demonstration provided in this paper is elegant and convincing.

The deeper question is whether or not both the depression and the psychosocial impairment arise from common risk factors or whether the depression "causes" (at least in part) the psychosocial deficits. In other words, will treating depression more effectively or sooner decrease these psychosocial impairments or do we need to address them in a different fashion? Ultimately this question is not completely answered or answerable by this data. The good news is that adolescent MDD alone, when other factors (adolescent comorbidity, young adult psychopathology, current depressive symptoms, etc) were co-varied out, did not predict psychosocial impairment. To oversimplify that analysis, a single episode of depression in adolescence did not appear to lead to significantly worse young adult functioning. However, as depression is, in many individuals, a recurrent disorder, this gives only modest comfort.

At the very least, in addition to further improving our treatments for adolescent major depression, this work suggests that treatment that targets long term psychosocial impairment may be as necessary as treatment that targets the depressive syndrome itself.

Neal D Ryan, MD Joaquim Puig-Antich Professor in Child and Adolescent Psychiatry, University of Pittsburgh School of Medicine, USA 